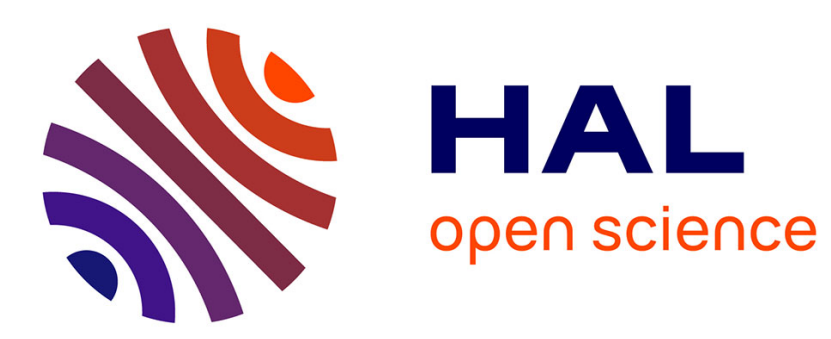

\title{
Coordination of Tilt and Touch in One- and Two-Handed Use
}

Theophanis Tsandilas, Caroline Appert, Anastasia Bezerianos, David Bonnet

\section{To cite this version:}

Theophanis Tsandilas, Caroline Appert, Anastasia Bezerianos, David Bonnet. Coordination of Tilt and Touch in One- and Two-Handed Use. Proceedings of the SIGCHI Conference on Human Factors in Computing Systems, Apr 2014, Toronto, Canada. pp.2001-2004, 10.1145/2556288.2557088 . hal00989343

\section{HAL Id: hal-00989343 \\ https://hal.science/hal-00989343}

Submitted on 10 May 2014

HAL is a multi-disciplinary open access archive for the deposit and dissemination of scientific research documents, whether they are published or not. The documents may come from teaching and research institutions in France or abroad, or from public or private research centers.
L'archive ouverte pluridisciplinaire HAL, est destinée au dépôt et à la diffusion de documents scientifiques de niveau recherche, publiés ou non, émanant des établissements d'enseignement et de recherche français ou étrangers, des laboratoires publics ou privés. 


\section{Coordination of Tilt and Touch in One- and Two-Handed Use Theophanis Tsandilas $^{1,2}$ Caroline Appert $^{2,1} \quad$ Anastasia Bezerianos $^{2,1} \quad$ David Bonnet $^{2,1}$ fanis@lri.fr $\quad$ appert@lri.fr $\quad$ anab@lri.fr $\quad$ bonnet@lri.fr

\author{
Inria $^{1}$ - Univ Paris-Sud \& CNRS $^{2}$ (LRI) \\ F-91405 Orsay, France
}

\begin{abstract}
Our goal is to enhance navigation in mobile interfaces with quick command gestures that do not make use of explicit mode-switching actions. TilTouch gestures extend the vocabulary of navigation interfaces by combining motion tilt with directional touch. We consider sixteen directional TilTouch gestures that rely on tilt and touch movements along the four main compass directions. An experiment explores their effectiveness for both one-handed and two-handed use. Results identify the best combinations of TilTouch gestures in terms of performance, motor coordination, and user preferences.
\end{abstract}

\section{Author Keywords \\ Mobile devices; Tilt; Touch; Gestures; Touchscreen;}

\section{ACM Classification Keywords}

H.5.2 [Information Interfaces and Presentation]: User Interfaces - Graphical user interfaces;

\section{INTRODUCTION}

Motion sensors such as accelerometers and gyroscopes offer a new space for the design of mobile interaction techniques that combine touch and tilt. Several previous approaches $[1,13]$ have studied motion-based gestures, but they depend on recognizers of rather exaggerated movements or explicit modeswitching techniques. We address this limitation by combing the two modalities, touch and tilt, as part of a single TilTouch gesture. TilTouch gestures do not interfere with simple touch and tilt actions. Thus, users can activate them within the flow of common navigation tasks without having to perform additional actions to switch between modes.

Our approach lies within the Sensor Synaesthesia design space [6], but we focus on directional drags rather than static touch. We empirically study the effectiveness of TilTouch gestures in terms of both user performance and preferences. We also investigate the extent to which users can control tilt and touch as integral input dimensions [7]. We study the use of the gestures for both one and two hands as each use is subject to different symmetries and requires the coordination of different limbs. We show that the same set of TilTouch gestures can be effective for both one- and two-handed use.

Theophanis Tsandilas, Caroline Appert, Anastasia Bezerianos, and David Bonnet. Coordination of Tilt and Touch in One- and Two-Handed Use. In CHI'14: Proceedings of the 32nd International Conference on Human Factors in Computing Systems, 2001-2004, ACM, April 2014.

(C)ACM, 2014. This is the author version of the work. It is posted here by permission of ACM for your personal use. Not for redistribution. The official version was published in $\mathrm{CHI}$ 2014, April 26 - May 01 2014, Toronto, ON, Canada. http://dx.doi.org/10.1145/2556288.2557088.

\section{RELATED WORK}

We can distinguish between two main forms of touch gestures for activating commands in mobile interfaces: free-form gestures, such as alphabetical ones [9], and directional marks inspired by marking menus [8]. According to Bragdon et al. [3], simple marks are more accurate than free-form gestures.

Tilt input has been used for navigation in sequential lists, twodimensional spaces, and menus containing few items, typically three to four [10, 12]. Rahman et al. [11] have empirically studied the number of possible subdivisions along each main tilt direction around the wrist. They found that error rates are never below $5 \%$ on average, even for the smallest sets (four items) that they considered, and increases fast with the size of the set. Other projects considered more elaborated motion gestures like DoubleFlip [13] and JerkTilt gestures [1]. In the newest mobile devices, tilting automatically adjusts the view orientation of a device to portrait or landscape to fit its physical orientation $[2,5]$.

The Sensor Synaesthesia design space [6] combines tilt and touch to create richer interaction sequences. Specifically, it proposes tilt and touch interactions for discrete or continuous commands, however touch remains at a fixed screen location. The work also only reports on subjective feedback from an informal user evaluation. Thus, combined tilt and touch has never been studied before through a formal experiment.

\section{COMBINING TOUCH WITH TILT}

Our goal is to facilitate command invocation while users perform navigation tasks. By combining drags and tilts, we can enhance the number of available gestures but also avoid the use of explicit mode-switching. To that end, we explored how a TilTouch gesture should be defined such that it does not interfere with existing navigation gestures. We conducted a preliminary study to determine the angular range of unintentional tilts that occur during common drag and swipe actions. 10 participants interacted with three representative mobile interfaces: a large 2D view, a vertical list of 50 items, and a horizontally aligned view of five screens. We analyzed a total of 3935 events of navigation or directional-drag gestures and defined unintentional relative tilt angles to be in the range of $[-18,18]$ degrees, where tilts are measured relatively to the initial orientation of the phone when the finger starts touching the screen. This range corresponds to less than $0.2 \%$ of false positives and does not hinder the activation of intentional TilTouch gestures. We also set the minimum drag distance for activating a gesture to $7 \mathrm{~mm}$. When these criteria are met, we detect the dominant direction of the tilt (tiltDir) and the drag (dragDir) to recognize the TilTouch gesture $\langle t i l t D i r, d r a g D i r\rangle$. The command associated with this combination is executed after lifting the finger. 


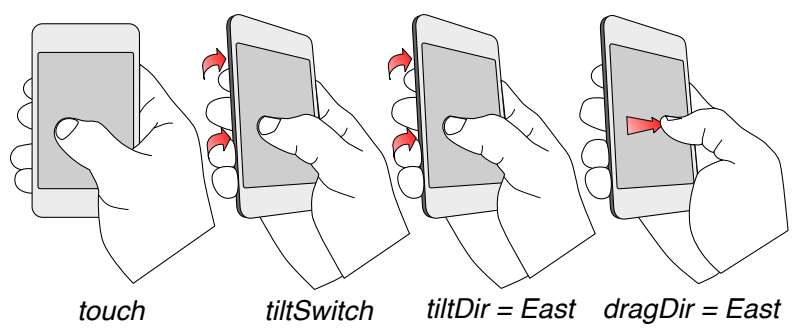

Figure 1. $\langle$ tiltDir, dragDir $\rangle=\langle$ East, East $\rangle$ gesture performed with one hand. Here, the user starts with a tilt followed by a touch action.

Our goal is to keep the shape of gestures as simple as possible while providing a reasonable number of accessible commands. Therefore, we only consider the simplest touch marks and tilt angles along the four cardinal directions: North, South, East, and West. Combining such simple gestures along the touch and tilt modality results in a total of 16 commands. Figure 1 illustrates an example where the user performs an $\langle$ East, East $\rangle$ gesture.

TilTouch gestures are especially useful as quick triggers of commands, e.g., copy-paste, make a call, and add a bookmark on a map. The technique requires the sequential or parallel control of two input modalities. We envision that novice users will start discovering the gestures by controlling tilt and touch in sequence. However, we expect that with practice, expert users will be able to internalize a single action and use the two input channels in parallel, combining the two gestures into a single interaction chunk [4]. As other directional gestures, TilTouch gestures have drawbacks: drags are limited near screen edges, and screen visibility is reduced both by the finger and the tilt. Yet, our low threshold value of relative tilts $\left( \pm 18^{\circ}\right)$ minimizes this problem.

\section{EXPERIMENT}

We conducted a lab study where participants performed TilTouch gestures in conjunction with regular drags. Our goals were to find: (1) which TilTouch gestures are more effective; (2) whether the same gestures are appropriate for both single and two-handed use; and (3) whether users can control the two input modalities in parallel.

\section{Participants and Apparatus}

24 volunteers ( 7 women), 22 to 45 years old participated in the experiment. 23 were right-handed, one was mixedhanded. Participants interacted with a 4.3-inch $480 \times 800$ pixel Samsung Galaxy S2, running our software implemented in Java under Android 2.3.3.

\section{Task and Conditions}

The experimental task consisted of two sub-tasks:

Pre-task (Figure 2-(a-b)). First, the user drags a ring of 160pixel diameter over another 40-pixel target ring. The direction of this sliding movement randomly varied circularly around the center of the screen. The pre-task represents a usual drag navigation task that interlaces with TilTouch gestures.

Main task (Figure 2-(c-d)). Then, the user completes the main task by performing a TilTouch gesture. As shown in Figure

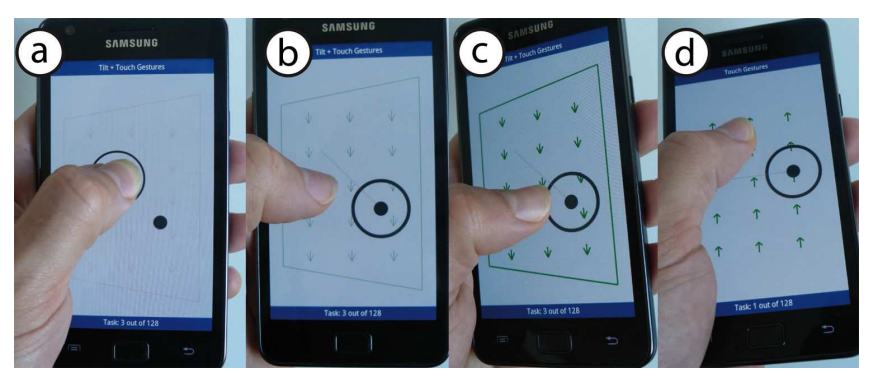

Figure 2. An experimental task for directional-tilt gestures. (a) Pre-task: The user starts sliding a ring over a circular target; (b) The pre-task has been completed; (c) Main task: The user tilts the device to the indicated direction while dragging the finger. (d) Main task for free-tilt gestures: visual instructions are only provided for the direction of the drag.

2 , instructions about the target direction of the tilt and drag movements are constantly displayed on the screen. The drag direction is indicated with a flock of arrows while the tilt direction is shown with a trapezoid frame that represents the view of the device in perspective.

To assess the overhead due to selecting a given tilt direction, we tested two types of gestures:

Free Tilt. Participants were asked to perform a directional drag according to a suggested direction in conjunction with a tilt in any tilt direction. No tilt direction was indicated.

Directional Tilt. Participants were asked to perform both a directional drag and a directional tilt according to a suggested combination of TilTouch directions. We tested all the 16 possible settings of combined cardinal directions, e.g., tilting to the North and dragging to the West.

We also tested the two typical mobile uses:

One hand. Participants completed the tasks with their right hand and used their thumb to touch the screen. The tilt and the drag movement were both performed by the same hand.

Two hands. Participants hold the device with their left hand but used the index finger of their right hand to touch the screen. The tilt movement was performed by the left hand while the drag movement was performed by the right hand.

\section{Design, Procedure and Measures}

We followed a mixed full-factorial design, with mobile use being a between-participants factor, and the remaining variables as within-participants factors. We analyzed 6144 tasks:

2 mobile uses $\times 12$ participants

$\times 2$ types of gestures $\times 8$ blocks

$\times 4$ drag directions $\times 4$ tilt directions

In addition to these tasks, each participant completed 24 practice tasks for each type of gestures. The tasks were grouped into eight blocks of 16 tasks ( 4 drag directions $\times 4$ tilt directions) presented in a random order. As no tilt direction is enforced for free tilt gestures, each participant performed four replications of each drag direction in each block.

Before the beginning of the session, participants filled out a pre-study questionnaire eliciting demographic information 


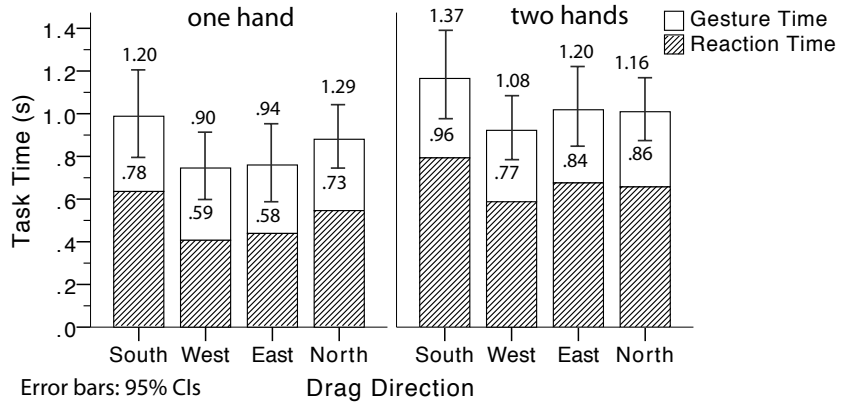

Figure 3. Free Tilt: Effect of drag direction

and their experience with smartphones and touch-enabled mobile devices. They were asked to interact in a standing position and always use their preferred hand. After the end of the session, participants answered a post-study questionnaire. They were asked to evaluate each of the 16 combinations of tilt and touch directions by using a 5-level Likert scale. The whole procedure lasted 30 to 40 minutes.

We measured the Task Time to complete the main task, which includes the Reaction Time to prepare a gesture and the Gesture Time to execute it. We considered two errors: accidental activations of TilTouch gestures during the first subtask (PreTask Error), and activations of the wrong direction, either tilt or drag (Gesture Error). In both cases, the user had to restart.

\section{Results}

For the analysis of the time measures, we conducted ANOVAs with mobile use treated as a between-participants factor, and type of gesture, block, drag direction and tilt direction treated as repeated measures. For the analysis of errors and user ratings, we used non-parametric tests.

Effect of Mobile Use and Type of Gesture. The difference for the overall Gesture Time was not significant between the two mobile uses $(p=.65)$. However, taking a closer look reveals that participants had a higher Reaction Time $\left(F_{1,22}=6.73\right.$, $p=.017$ ) in a two-hand use. This indicates a higher amount of planning to coordinate control.

A comparison between free-tilt and directional-tilt gestures shows that constraining tilt direction has a great cost to user performance. This cost was significant for both Reaction Time $\left(F_{1,22}=27.18, p<.001\right)$ and Gesture Time $\left(F_{1,22}=\right.$ 10.03, $p=.004)$. Similarly, Gesture Error was higher for Directional Tilt $(Z=-4.2, p<.001)$, raising from $1.0 \%$ $[0.5 \%, 1.6 \%]$ to $8.4 \%[6.6 \%, 10.2 \%]^{1}$. A $71 \%$ of the latter errors were due to incorrect tilts. Interestingly, the error rate was lower for two-handed use $(Z=-2.03, p=.045)$. Considering only directional tilts, it was $10.5 \%[8.0 \%, 13.1 \%]$ for one hand and $6.3 \%[3.7 \%, 8.8 \%]$ for two hands. PreTask Error was generally low, with a mean value of .5\% [.0\%, 1.1\%].

Effect of Drag and Tilt Direction. We first examine users' strategies for Free Tilt. For $80 \%$ of the tasks executed with one hand and $67 \%$ of the tasks executed with two hands, the direction of tilt and drag was the same. However, this strategy

\footnotetext{
${ }^{1}$ We report $95 \%$ confidence intervals (CIs) of the means in brackets.
}

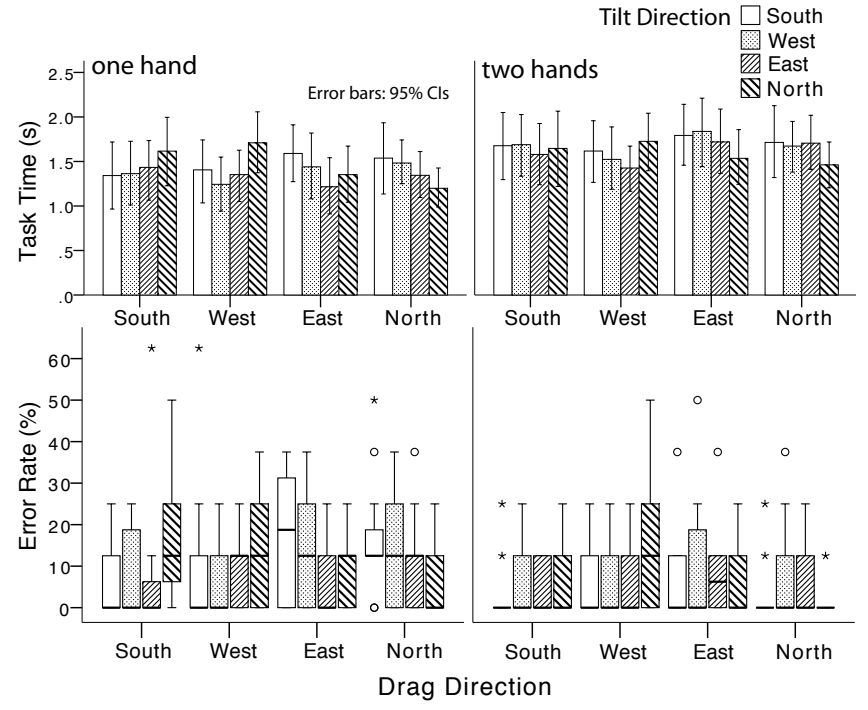

Figure 4. Directional Tilt: Task Time and Gesture Error for the 16 TilTouch combinations. Circles and asterisks in the box plots represent mild and extreme outliers, respectively. Dark lines represent medians.

was not consistent among all the participants. One participant (one hand) almost always chose an East tilt. A second (two hands) always tilted South, while a third (two hands) always tilted North. Three other participants (two hands) used a North tilt for their South drags.

For Free Tilt, the dragging direction had a significant effect on Task Time $\left(F_{3,66}=6.18, p=.001\right)$. As shown in Figure 3, the West direction was the fastest and the South direction was the worst. We found no significant interaction between dragging direction and conditions $(p=.74)$. Differences were consistent for both one hand and two hands. Pairwise comparisons with Bonferroni's correction showed a significant difference between West and South $(p<.001)$ and between East and South ( $p=.021)$. Differences were mostly due to reaction times. Our interpretation is that planning the tilt for the South drag direction is possibly harder.

For Directional Tilt, we did not observe any effect of drag $(p=.65)$ or tilt direction $(p=.18)$ on Task Time. The effect of their interaction was significant though $\left(F_{4.5,99.1}=\right.$ $2.95, p=.003)$. Figure 4 shows the detailed results for the two mobile uses. By inspecting both task times and error rates, we see that certain tilt and drag combinations were particularly problematic: $\langle$ North, West $\rangle$ for both conditions, and $\langle$ North, South $\rangle,\langle$ South, East $\rangle,\langle$ West, East $\rangle$, $\langle$ South, North $\rangle$ and $\langle$ West, North $\rangle$ for one hand. In corroboration with observations on free-tilt gestures, combinations with same tilt and drag directions had the best performance. This was especially true for one-handed use. Overall, North tilts were more compatible with North and East drags, while South tilts were with South and West drags.

Subjective Ratings. The tilt direction had a significant effect on participants' rating $\left(\chi^{2}(3)=15.61, p=.001\right)$. Pairwise comparisons with adjusted $p$ values showed that East tilts received a significantly higher score than West 

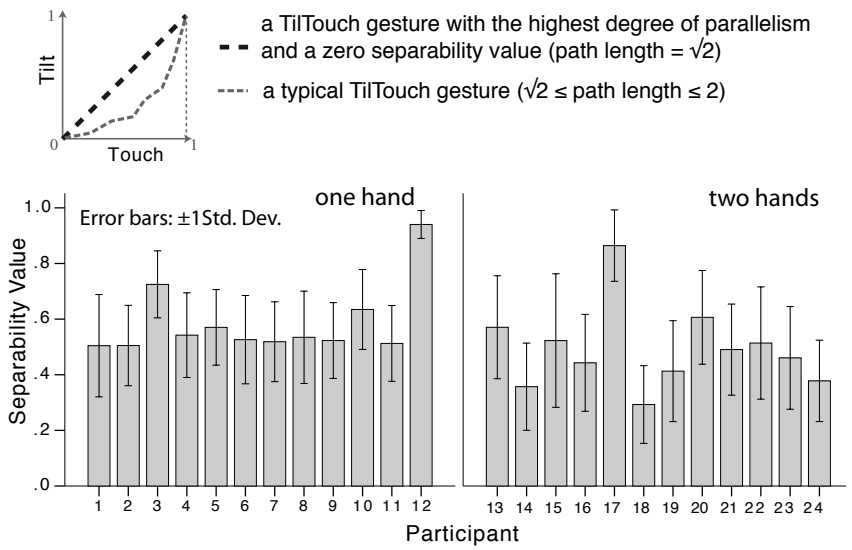

Figure 5. The normalized TilTouch space (top). Separability values for each participant (bottom). Presented only for directional tilts.

tilts $(p=.003)$. West tilts generally received low ratings for two hands as opposed to East tilts, which were highly ranked. The drag direction did not have any significant effect on ratings $\left(\chi^{2}(3)=4.26, p=.24\right)$, but the effect of the combined tilt and drag direction was significant $\left(\chi^{2}(15)=100.43, p<.001\right)$. Participants gave high ratings to gestures with the same tilt and drag direction. Interestingly, participants using two hands rated $\langle$ East, West higher than $\langle W e s t, W e s t\rangle$. Other gestures received low scores for both conditions: $\langle$ North, West $\rangle,\langle$ South, East $\rangle$, $\langle$ South, West $\rangle,\langle$ West, South $\rangle$, and $\langle$ West, North $\rangle$. Surprisingly, $\langle$ North, South $\rangle$ was highly rated under one-hand use despite its poor performance. Overall, participants considered opposite tilt and drag directions easy to perform.

Degree of Parallelism. We analyzed the degree of parallelism of the tilt and touch input channels. To that end, we plotted the collected TilTouch gestures in the normalized tilt+touch space shown in Figure 5 (top). The path length of a TilTouch gesture exhibiting the highest degree of parallelism is $\sqrt{2}$ while a TilTouch gesture with no parallel control has a length of 2. By normalizing the path length of each TilTouch gesture, we obtain the Separability measure $\in[0,1]$ : the less parallel the control over the two input channels the higher the value of Separability.

Figure 5 shows its distribution across participants for directional tilts. Its mean value ranged from .29 (Participant 18) to .94 (Participant 12), who executed the tilt and the drag gesture always in sequence. We found a positive correlation between mean Separability and mean GestureTime (Pearson correlation $=.621, p=.001)$. The mean value of Separability was $.59[.49,70]$ for one hand and $.49[.40,59]$ for two hands, but this difference was not statistically significant $\left(F_{1,18}=2.20\right.$, $p=.16)$. We found a significant effect of both drag direction $\left(F_{3,54}=3.36, p=.025\right)$ and tilt direction $\left(F_{3,54}=3.6\right.$, $p=.023)$ on Separability. Their interaction effect was also significant $\left(F_{4.6,82.3}=4.73, p=.001\right)$. Pairwise comparisons with Bonferroni's correction showed that West drags resulted in significantly more parallel movements than North drags $(p<.05)$. Finally, we observed low separability values for tilts and drags of the same direction for both conditions.

\section{DISCUSSION AND CONCLUSIONS}

We have studied combined touch and tilt gestures for oneand two-handed interaction with mobile phones. Our findings suggest that most users can control the two input modalities in parallel ( $40-50 \%$ of optimal parallelism) under both mobile uses. We observed that not all combinations of tilt + touch are effective. TilTouch gestures along the same direction result in lower error rates, are more intuitive, and are highly preferred by users. East tilts were highly ranked. Finally, we found that North tilts were more compatible with North and East drags, while South tilts with South and West drags. These results are consistent between one- and two-handed use. This is an important finding suggesting that designers can use the same set of usable TilTouch gestures for both hand configurations. Future work needs to explore appropriate visual guides that assist in the discovery and effective use of such gestures.

Acknowledgments. This work was partly funded by ANR-11-JS02-004-01.

\section{REFERENCES}

1. Baglioni, M., Lecolinet, E., and Guiard, Y. Jerktilts: using accelerometers for eight-choice selection on mobile devices. ICMI '11, ACM (2011), 121-128.

2. Bartlett, J. Rock'n'scroll is here to stay. Computer Graphics and Applications, IEEE 20, 3 (2000), 40-45.

3. Bragdon, A., Nelson, E., Li, Y., and Hinckley, K. Experimental analysis of touch-screen gesture designs in mobile environments. CHI '11, ACM (2011), 403-412.

4. Buxton, W. A. S. Human-computer interaction. Morgan Kaufmann Publishers Inc., San Francisco, CA, USA, 1995, ch. Chunking and phrasing and the design of human-computer dialogues, 494-499.

5. Hinckley, K., Pierce, J., Sinclair, M., and Horvitz, E. Sensing techniques for mobile interaction. UIST '00, ACM (2000), 91-100.

6. Hinckley, K., and Song, H. Sensor synaesthesia: touch in motion, and motion in touch. CHI'11, ACM (2011), 801-810.

7. Jacob, R. J. K., Sibert, L. E., McFarlane, D. C., and Mullen, Jr., M. P. Integrality and separability of input devices. ACM Trans. Comput.-Hum. Interact. 1, 1 (1994), 3-26.

8. Kurtenbach, G., and Buxton, W. User learning and performance with marking menus. In Proc. CHI '94, ACM (1994), 258-264.

9. Li, Y. Gesture search: a tool for fast mobile data access. UIST '10, ACM (2010), 87-96.

10. Oakley, I., and Park, J. A motion-based marking menu system. CHI EA '07, ACM (2007), 2597-2602.

11. Rahman, M., Gustafson, S., Irani, P., and Subramanian, S. Tilt techniques: investigating the dexterity of wrist-based input. CHI '09, ACM (2009), 1943-1952.

12. Rekimoto, J. Tilting operations for small screen interfaces. UIST'96, ACM (1996), 167-168.

13. Ruiz, J., and Li, Y. Doubleflip: a motion gesture delimiter for mobile interaction. CHI'11, ACM (2011), 2717-2720. 